\title{
An annotation and modeling schema for prescription regimens
}

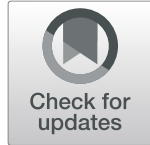

John Aberdeen, Samuel Bayer, Cheryl Clark, Meredith Keybl and David Tresner-Kirsch ${ }^{*}$ (1)

\begin{abstract}
Background: We introduce TranScriptML, a semantic representation schema for prescription regimens allowing various properties of prescriptions (e.g. dose, frequency, route) to be specified separately and applied (manually or automatically) as annotations to patient instructions. In this paper, we describe the annotation schema, the curation of a corpus of prescription instructions through a manual annotation effort, and initial experiments in modeling and automated generation of TranScriptML representations.

Results: TranScriptML was developed in the process of curating a corpus of 2914 ambulatory prescriptions written within the Partners Healthcare network, and its schema is informed by the content of that corpus. We developed the representation schema as a novel set of semantic tags for prescription concept categories (e.g. frequency); each tag label is defined with an accompanying attribute framework in which the meaning of tagged concepts can be specified in a normalized fashion. We annotated a subset (1746) of this dataset using cross-validation and reconciliation between multiple annotators, and used Conditional Random Field machine learning and various other methods to train automated annotation models based on the manual annotations. The TranScriptML schema implementation, manual annotation, and machine learning were all performed using the MITRE Annotation Toolkit (MAT). We report that our annotation schema can be applied with varying levels of pairwise agreement, ranging from low agreement levels $(0.125 \mathrm{~F}$ for the relatively rare REFILL tag) to high agreement levels approaching $0.9 \mathrm{~F}$ for some of the more frequent tags. We report similarly variable scores for modeling tag labels and spans, averaging 0. 748 F-measure with balanced precision and recall. The best of our various attribute modeling methods captured most attributes with accuracy above 0.9 .

Conclusions: We have described an annotation schema for prescription regimens, and shown that it is possible to annotate prescription regimens at high accuracy for many tag types. We have further shown that many of these tags and attributes can be modeled at high accuracy with various techniques. By structuring the textual representation through annotation enriched with normalized values, the text can be compared against the pharmacist-entered structured data, offering an opportunity to detect and correct discrepancies.
\end{abstract}

Keywords: Medication, Annotation, Prescriptions, Modeling

\section{Background}

Patient medication regimens are described in a variety of genres of medical documents, including prescription orders, intake interview medication lists, discharge summaries, prescribing guidelines, and medication orders. Often, at least some aspects of the regimen are described in free text; in many cases, the entire regimen is specified in free text alone. Regimen information is essential to patient care, as well as for secondary uses such as

\footnotetext{
* Correspondence: davidtk@mitre.org

The MITRE Corporation, 202 Burlington Rd, Bedford, MA 01730, USA
}

retrospective studies and pharmacovigilance, but the free text representation presents great challenges in accessing the information computationally. In this introduction we describe the availability and current state of the art of medication information extraction tools. We then describe community evaluations, open representation schemata, and corpus development efforts in the medication regimen domain.

\section{Medication information extraction systems}

Over the last two decades, several systems have been developed to identify medication names and associated

(c) The Author(s). 2019 Open Access This article is distributed under the terms of the Creative Commons Attribution 4.0 International License (http://creativecommons.org/licenses/by/4.0/), which permits unrestricted use, distribution, and 
dosage attribute information in the free text of clinical reports. Early rule-based systems include CLARIT [1], MedLEE [2, 3], and MERKI [4]. MERKI is an open source system that uses a library of regular expressions and a lexicon of drug names to identify medication names and dosage attributes. Authors of this system report accuracies of $83.7 \%$ for dose, $88.0 \%$ for route of administration, and $83.2 \%$ for frequency. CLARIT, a commercial system, combines basic NLP, general and special lexicons, and pattern matching rules to identify medication names and dosage attributes. MedLEE, a commercial system developed to extract various medical concepts, identifies medication names but not dosage attributes. Additional commercial systems include
LifeCode $^{\text {tx }}$ from A-Life Medical, Inc., Natural Language Patient Record ${ }^{\mathrm{Tm}}$ from Dictaphone Corporation, and FreePharma $^{\text {Tw }}$ from Language and Computing NV. Algorithms for these systems are not publicly available.

A 2009 assessment of the medication extraction performance of commercial systems from four vendors (Language and Computing, Coderyte, LingoLogics, and Artificial Medical Intelligence) [5] found that they did well identifying medication names (F-measure 0.932) but less well identifying attributes such as strength $(\mathrm{F}=0.853)$, route $(\mathrm{F}=0.803)$, and frequency $(0.483)$, and concluded that automated extraction could support but not replace a manual process for clinical applications such as medication list generation.

Table 1 A comparison of concept coverage, and the identifiers for those concepts, in various information representations: MedXN/ PredMed information extraction output, SHARPn annotation schema, FHIR clinical data structures, and TranScriptML

\begin{tabular}{|c|c|c|c|}
\hline MedXN / PredMed & SHARPn & FHIR & TranScriptML \\
\hline \multicolumn{4}{|c|}{ Dosage: amount of medication to be taken with each administration } \\
\hline Dosage & Dosage & Dose & Take, Doseamount \\
\hline \multicolumn{4}{|c|}{ Duration: how long patient is expected to be or has been taking the drug } \\
\hline Duration & Duration & & Duration \\
\hline \multicolumn{4}{|c|}{ Form: Physical form of the drug } \\
\hline Form & Form & & Form \\
\hline \multicolumn{4}{|c|}{ Frequency: how often the drug should be administered } \\
\hline Frequency & Frequency & Frequency, frequencyMax, period, periodMax, periodUnits & Freq \\
\hline
\end{tabular}

Indication: the reason the drug is being taken by the patient

Reason

Indication

Medication: Name of the drug

Medication

Medication

Medication

Medication

Miscellaneous:

Modifiers

Additional Instructions

Instruction

PRN: Whether the drug is to be taken as needed

$$
\text { asNeeded }
$$

PRN

Route: how the drug is administered

Route Route

Route

Route

Status: whether the medication is currently being taken

$$
\text { Status Change }
$$

Strength: the amount of active drug per unit (e.g. per tablet or per ml solution)

Strength Strength 
The i2b2 2009 Medication Challenge shared task [6] focused on extraction of medication-related information from clinical text. The information to be extracted included medication name, dosage amount, route of administration, frequency, duration, and reason for administration. Twenty teams participated in this challenge, and while all of the top 10 systems recognized medication names well with F-measures above 0.75 F-measure, they performed less well on other attributes. The attributes that proved hardest to extract were durations and reasons, for which the highest scores were 0.525 and 0.459 , respectively.

Seven of the top ten performing systems were rule-based systems [7-13]. Three of the top ten [14-16] were hybrid systems that combined machine learning and rules, including the highest ranking system [14], which used machine learning for tagging and rules for integrating related components.

PredMed [17] and MedXN [18] are two more recent systems which improve on the accuracy demonstrated by the 2009 i 2 b2 challenge entries. PredMed is not yet publicly available; MedXN is available as a free and open-source UIMA-based tool. Both target the same set of seven medication-related concepts, which are listed in Table 1 in comparison to other information representations.

Both PredMed and MedXN find spans referencing these seven concept types in text. Additionally, MedXN assigns an RxCUI id to normalize the medication name, performs coreference between medication names and regimen concepts, and attempts to assign an RxCUI normalization to the full medication concept. The full normalization produces a structured string combining the referenced regimen concepts. However, neither system normalizes the individual concepts (e.g. Frequency); individual concept references are left in their original surface text form.

\section{Medication annotation schemas}

In the i2b2 2009 challenge, the target output included standoff annotations of six fields of medication information (medication names, doses, modes [i.e. routes], frequencies, durations, and reasons [i.e. indications]). This schema captures the text positions and surface text of each category of information, but does not capture any semantic or normalized representation for each tagged instance.

A large annotation task undertaken by Strategic Health IT Advanced Research Projects (SHARP) Research Focus Area 4 (SHARPn) consisted of annotating a variety of medical named entities in clinical notes. The annotation task was intended to support development of clinical NLP tools. The SHARPn NLP team used the annotation to improve the functionality, interoperability, and usability of a clinical NLP system, Clinical Text Analysis and
Knowledge Extraction System (cTAKES), which is now publicly available as Apache cTAKES (http://ctakes.apache.org/).

The SHARPn annotation task consisted of (1) identifying mentions of clinical concepts (i.e. spans of source document text which refer to those concepts), including medications, (2) mapping them to a UMLS code [19] from the provided terminology (RxNORM for medications) [20], and (3) identifying modifiers or attributes of the mention. Terms to be annotated as a medication were terms belonging to a specified set of UMLS semantic types with RxNORM as the terminology source. In the SHARPn annotation task, the annotation was applied to a corpus of free-text clinical notes, including radiology and breast cancer notes, in which medication mentions occur primarily in sentential text or semi-structured text such as medication lists [21, 22].

SHARPn's annotation types related to medication regimens are listed in Table 1 in comparison to other information representations; an additional annotation, Allergy_Indicator, relates to medications, but not to prescribed regimens. In addition to medication-specific attributes, several general attributes (that is, attributes not specific to a particular entity class) were applied to medication text: Negation_indicator, Uncertainty_indicator, Conditional, Subject, and Generic.

The SHARPn schema captures text positions and surface text for medication names and attributes, and normalized representation of medication names with RxNorm codes. Normalization of dosage attributes was not a focus of the annotation effort, and reasons for taking a drug (i.e., indication) were not included as part of the medication annotation task.

A 2015 BioNLP effort [23] captured annotations of medication information from Adverse Event Report documents. In addition to adverse event content, these annotations captured medication names as well as several types of regimen information: Dosage, Route, Frequency, and Duration. However, normalization of the captured information was not within the scope of this effort.

\section{FHIR medication resource schema}

The medication information representation schema referenced above all relate specifically to inline annotation of medication regimen concepts, and the information extraction systems described have been designed and evaluated in the context of those annotation schema. To fulfil the promise of NLP-enabled downstream applications such as medication decision support and medication reconciliation, information extraction systems must produce results that are compatible with the information structures used by EHRs and other production systems. A full survey of clinical applications' schemas for representing medication 
information is out of scope of this article, but TranScriptML's attribute structure for normalizing regimen concepts was designed to be compatible with the Fast Healthcare Interoperability Resources (FHIR) standard representation.

Health Level Seven (HL7) is currently developing FHIR, a standard for RESTful exchange of clinical data [24]. FHIR is not an annotation schema and is not intended as a markup language for natural language data, but it is relevant for its inclusion of a richly detailed data structure for medication regimens in its MedicationOrder resource. FHIR MedicationOrders [25] include, among other data, regimen fields related to dosage, frequency (highly structured and allowing for normalization of expressions like "take $\mathrm{X}$ to $\mathrm{Y}$ times per $\mathrm{Z}$ days, with meals"), indication, and route. Table 1 shows these data types in comparison to other representations.

\section{Methods}

\section{Description of the data}

We developed our annotation schema and conducted our experiments using a dataset obtained from Partners Healthcare. The full dataset consisted of 2914 prescriptions, each of which included a number of fields that contain structured data (e.g., ID, medication, dose, form, frequency, duration, etc.) as well as a directions field containing unstructured text (e.g., "take 3 tablets twice a day for the next 2 weeks then stop"). Forty percent of the records were preserved as an unexamined test set for other related work, and 60\% (1746 records) were used in the

Table 2 Sample Prescription Record

\begin{tabular}{ll}
\hline FieldName & Contents \\
\hline ID & 247 \\
Medication & IBUPROFEN \\
Route & PO \\
Dose & 600 \\
Dose Units & MG \\
Strength & $600 \mathrm{MG}$ \\
Take & 1 \\
Form & Tablet \\
Frequency & TID \\
PRN & 1 \\
PRN Reason & Pain \\
Duration & 30 \\
Duration Units & \\
Dispense Quantity & 90 \\
Dispense Quantity Units & Tablet(s) \\
Directions & take 2 tablets three times a day as \\
\hline
\end{tabular}

present study to develop and test the TranScriptML annotation schema. Our annotation effort focused exclusively on the directions field, with other fields informing the design of the annotation tag set. A simplified sample input record appears in Table 2.

The directions field was extracted from each of the training records to create 1746 short text files for annotation.

\section{Annotation and modeling environment}

We constructed our annotation schema, conducted our annotation, reconciled our results, and built our models using the MITRE Annotation Toolkit (MAT). (MAT is a generalization and extension of the MIST de-identification system [26].) Open-source installation files and full documentation of MAT are available at http://mat-annotation.sourceforge.net/. MAT provides a declarative language for specifying the details of an annotation task, including tag names, attributes, and relations, as well as annotation workflows. MAT also provides a facility for building predictive models (via machine learning) from and conducting experiments with annotated data. The model building component implements machine-learning algorithms including Conditional Random Fields span annotation and Maximum Entropy classification. A sample record being annotated in MAT appears in Fig. 1.

\section{Annotation Schema}

Throughout the remainder of the paper, we will use the following terms with specific meanings: a tag refers to an annotation denoting that a medication regimen concept is described in a particular part of a document; a label refers to the category to which the tagged concept belongs (e.g. DOSEFORM, DURATION); a span refers to the specific portion of the document (defined by start and end character indices) to which the tag applies; an attribute is an annotated property of a tag (specific to the label type) which by itself or in combination with other attributes assigns a normalized semantic representation of the tagged concept.

Our annotation schema, TranScriptML, is designed to provide flexible markup and representation of the regimens described in prescription directions. It was developed iteratively; each iteration included redundant annotation of small subsets of the corpus $(\sim 40$ documents) by four annotators (A1-A4) using candidate TranScriptML schema versions. Discrepancies and flagged issues were discussed by all four annotators together after each iteration, which served the dual purposes of refining the schema and resolving annotator misunderstandings prior to primary annotation (which is described later). Once the schema stabilized, all documents used in schema development were reannotated along with the remainder for the sake of corpus consistency. TranScriptML contains 


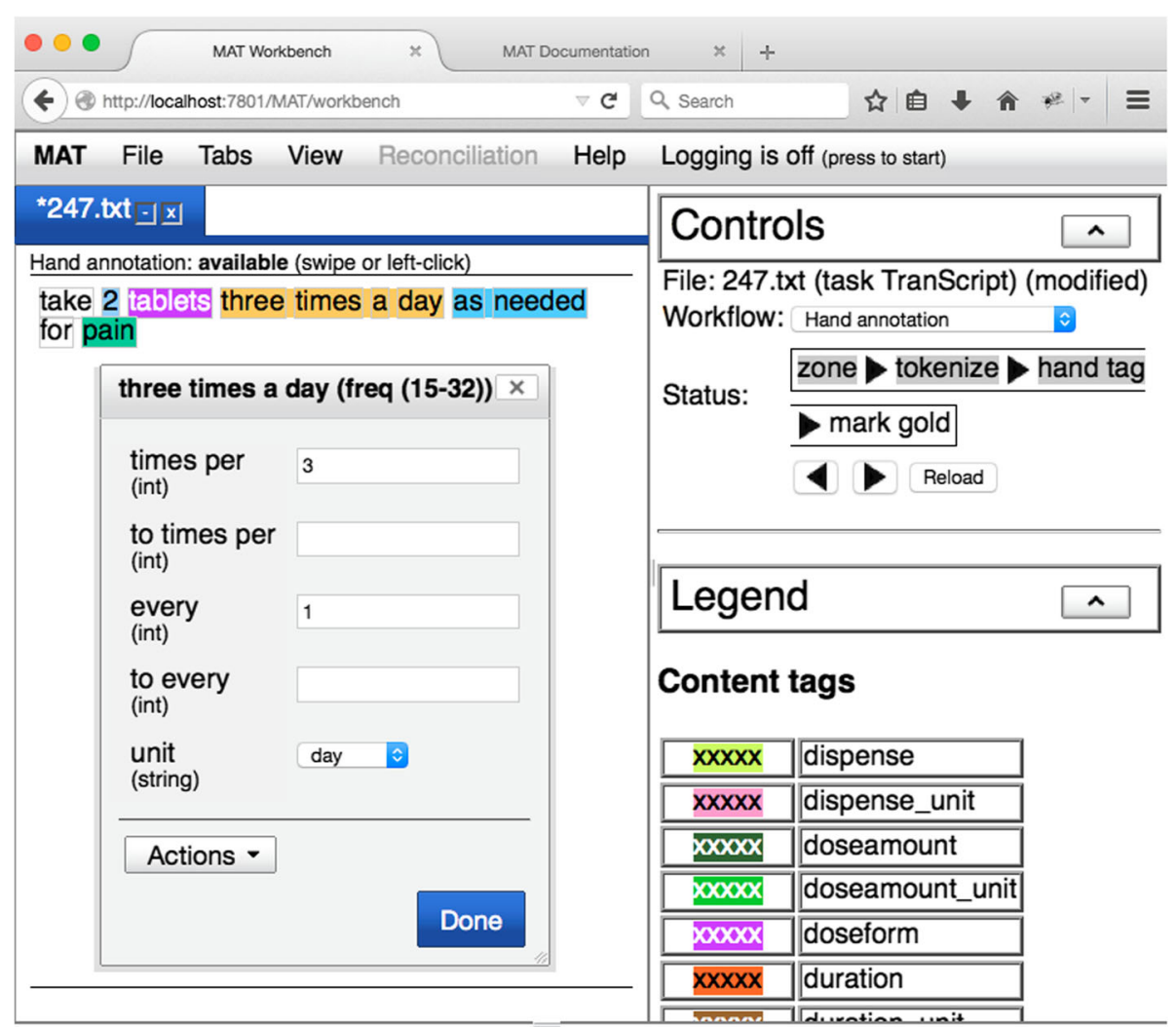

Fig. 1 Sample record in MAT annotation environment

19 tag types, each with associated attributes. TranScriptML is a detailed representation that expands significantly on the complexity of medication regimens that can be described by the schemata used in earlier representations such as those used in the i2b2 and SHARPn medication annotation challenges. For example, TranScriptML's attribute structure allows full specification of frequency ranges (e.g. "every 4-6 hours"), preserves the differences in meaning of frequency information that is stated as periods rather than frequencies (e.g. "every three days" vs. "three times per day"), and enables specification of additional timing information (e.g. "1 hour after meals"). The detailed attribute structure for dose, strength, frequency, and timing information is mappable to the detailed data structures used in FHIR's MedicationOrder resource, described earlier. The list of tag descriptions appears in Table 3.

There are several tag label types represented in TranScriptML. Simple span-only tags such as PRN and INDICATION mark spans of text that refer to corresponding concepts; these tags identify the concept spans but have no additional attributes to describe and normalize the content. Other tags have attributes associated with them that encode the semantics that the text spans describe. These attributes are either numeric (e.g., quantity of a
DOSEAMOUNT), text strings (e.g., units or events), or Boolean (e.g., REFILLs allowed or not allowed). Some tags are complex, with multiple attributes (e.g, FREQ and TIMING). A list of tags and their attributes appears in Table 4.

\section{Annotation effort}

Four annotators (A1-A4) participated in the study, and each document in the corpus was double-annotated. The 1746-document corpus was divided into 4 groups (G1- G4), with each annotator individually tagging all documents in 2 of the groups. After the initial annotation, each group of documents was adjudicated by a third annotator who had not been one of the primary annotators of that group. Finally, annotators A1 and A2 reconciled the entire corpus. This double-annotation, followed by the two-stage adjudication and reconciliation process was an effort to ensure we produced a consistently tagged corpus.

\section{Model building}

We conducted several learning experiments, described below, to model the annotations in our corpus. 
Table 3 TranScript annotation tag Label type descriptions

\begin{tabular}{|c|c|c|}
\hline Tag & Example & Description \\
\hline Dispense & 120 & The quantity of a medication to be issued by the pharmacist. \\
\hline Dispense_unit & tablets & \\
\hline Medication & Ibuprofen & Text specifying a specific pharmaceutical product. \\
\hline Take & 2 & The quantity of medication per application, from the patient's perspective. \\
\hline Strength & 400 & The amount of active ingredient per physical quantity of medication. \\
\hline Strength_unit & $\mathrm{mg}$ & \\
\hline Doseamount & 800 & The amount of active ingredient per application of medication. \\
\hline Doseamount_unit & $\mathrm{mg}$ & \\
\hline Doseform & capsules & The form of medication taken. Often marked in conjunction with TAKE. \\
\hline Duration & 3 & The period of time a patient should continue using a medication. \\
\hline Duration_unit & weeks & \\
\hline freq & 3-4 times per day & The frequency of use of a medication. \\
\hline Timing & before meals & $\begin{array}{l}\text { The intended timing of medication use or application. Differs from FREQ in } \\
\text { that it specifies temporal alignment of doses rather than patterns of repetition. }\end{array}$ \\
\hline PRN & as needed & Text indicating medication to be taken only as needed. \\
\hline Indication & pain & Condition for which medication is taken. \\
\hline Route & p.o. & A medication's manner or point of application to the body. \\
\hline Refill & no refills & Indicates whether refills are allowed or not. \\
\hline Sub_status & no substitutions & Indicates whether substitutions are allowed or not. \\
\hline Instruction & if pain persists, call your physician & Text indicating patient actions that are not captured by another tag. \\
\hline
\end{tabular}

We used the Carafe [27] Conditional Random Fields (CRF) engine included with the MAT distribution to learn models of tag spans and labels. For span tagging and labeling, we used MAT's default English tokenizer and the following feature set:

- Prefix and suffix ngrams of length up to 3

- Whether the current or previous token starts with a capital letter

- Whether the current token contains a digit

- The surface form of the current token

- The surface form of each of the single tokens 1,2 , or 3 tokens away from the current token

Depending on the intended use case of a trained model, the relative importance of precision and recall may not be equal, but rather there may be a particular need for high recall, or high precision. Carafe includes a parameter (prior_adjust) to adjust the tradeoff between precision and recall; we used this to build three span label models: one biased toward high recall (prior_adjust set to -3 ), one biased toward high precision (prior_adjust set to +3 ), and one with balanced recall and precision (default prior_adjust of 0 applied). Adjusting this parameter can result in higher recall at the expense of lower precision, or higher precision at the expense of lower recall.
For modeling the attributes of tagged spans, we used several different methods and combinations of methods, because there are several different types of attributes (numeric, string, Boolean), and many tags include multiple attributes of different types. We describe the attribute modeling methods below in the context of particular tags and classes of tags. Classifiers for modeling attributes were trained using Carafe's Maximum Entropy engine. Model building experiments for both span annotation and attribute learning used an 80/20 training/test split of the 1746-document annotated corpus.

\section{Preprocess}

Before modeling attributes, we normalize number expressions by using a numeric retokenizer that maps all number expressions to canonical forms. For example, three is mapped to 3 , one and a half to 1.5 , and 4 to 4 . Except where noted below, prescriptions containing normalized number expressions are the inputs for the modeling experiments.

\section{Frequency}

The FREQ tag is complex, encoding the times-per-day and/or timing interval for medications, as well as units for these numbers. As such, it contains both numeric and string attributes, and we explored several methods for modeling the attributes. For example, "take 2-3 times per day" would involve a FREQ tag with attributes 
Table 4 Transcript annotation tag label types and their attributes

\begin{tabular}{|c|c|c|}
\hline Tag & Attributes & Type (Values) \\
\hline Dispense & quantity, to_quantity & Numeric \\
\hline Doseamount & amount, to_amount & Numeric \\
\hline \multicolumn{3}{|l|}{ Strength } \\
\hline \multicolumn{3}{|l|}{ Take } \\
\hline Duration & num, to_num & Numeric \\
\hline Dispense_unit & unit & string (select from fixed list) \\
\hline \multicolumn{3}{|c|}{ Doseamount_unit } \\
\hline \multicolumn{3}{|l|}{ Strength_unit } \\
\hline Duration_unit & unit & string (minute, hour, day, week, month, other) \\
\hline Doseform & form & string (select from fixed list) \\
\hline Medication & name & string (free text defaults to text span) \\
\hline \multirow[t]{2}{*}{ Route } & route & string (select from fixed list) \\
\hline & side & string (left, right, both) \\
\hline \multirow[t]{2}{*}{ Freq } & $\begin{array}{l}\text { times_per, to_times_per, every, } \\
\text { to_every }\end{array}$ & numeric \\
\hline & unit & string (minute, hour, day, week, month, other) \\
\hline \multirow[t]{4}{*}{ Timing } & offset, to_offset & numeric \\
\hline & offset_unit & string (minute, hour, day, week, month, other) \\
\hline & direction & string (before, after, other) \\
\hline & event & $\begin{array}{l}\text { string (breakfast, lunch, dinner, meals, morning, noon, afternoon, evening, bedtime, } \\
\text { procedure, treatment, other) }\end{array}$ \\
\hline Instruction & note & string (free text input) \\
\hline Refill & refill & boolean \\
\hline Sub_status & subst & boolean \\
\hline PRN & (no attributes) & \\
\hline Indication & & \\
\hline
\end{tabular}

times_per $=2$, to_times_per $=3$, every $=1$, to_every $=$ null, and unit=DAY. The Baseline method simply counts each UNIT value and applies the most common value, along with the default numeric feature values of time$s \_p e r=1$, to_times_per $=$ null, every $=1$, and to_every $=$ null. The Hybrid method used a simple model containing bag of words, bigrams, and count of number expressions as features for learning the value of the UNIT attribute, as well as to determine whether the FREQ instance is an interval (every $\mathrm{N}$ time-units), or a count ( $\mathrm{N}$ times per time-unit) phrase. Our Classifier method is a variation on the Hybrid method that adds a classifier for each numeric attribute, normalizing to allow only $1-12$, $15,30,45$ and 60 as valid values.

\section{Timing}

The TIMING tag is also complex, with multiple numeric and string attributes. The Baseline method chooses the most common value for each attribute. The Hybrid method builds a classifier for direction, event, and offset_unit, and maps the numeric attributes directly from the normalized token list. The Classifier method adds a classifier for the numeric attributes as well, using the same features as used for the FREQ tag, and normalizing numeric attributes to the same list of valid values.

\section{Numeric attributes}

DISPENSE, DOSEAMOUNT, STRENGTH, TAKE, and DURATION all have only numeric attributes. We experimented with just two conditions here, Un-normalized, in which the source string is mapped as-is to the attribute (e.g., "take <TAKE amt='three'>three $</$ TAKE $>$ tablets"), and Normalized, in which the numeric retokenizer preprocess is applied (e.g., "take <TAKE amt='3'>three $<$ /TAKE $>$ tablets").

\section{Choice attributes}

Applying the unit tags (DISPENSE_UNIT, DURATION_UNIT, etc.) and ROUTE involves selecting attribute values from fixed lists. We explored four methods for modeling these attributes. The Baseline method simply chooses the most common value seen in the training 
data. The Literal Match method memorizes the mapping between text spans and attribute values in the training data, and uses the most common match. As a fallback, if a span does not appear in the training data, but matches one of the available values, the value is selected. The Levenshtein Fallback method is similar to the Literal match method, but the fallback method selects the value having the smallest edit distance [28] from the span, rather than requiring an exact match. Finally, the Classifier method builds a classifier for each attribute, using bag-of-words and bigrams as features.

\section{Boolean attributes}

For the Boolean attribute tags (REFILL and SUB_STATUS), our Baseline method chooses the most common value seen in the training data, and the Classifier method builds a classifier for each attribute using bag-of-words and bigrams as features.

\section{Results}

Pairwise agreement

Because each document was annotated by exactly two of the four annotators, we calculated pairwise agreement for each of the four annotator pairings (G1-G4) by calculating the F-measure between each set of annotations. These calculations are presented both broken down by tag label and also in total, and they reflect the degree of agreement between human annotators without reference to automated system output. Pairwise agreement results by F-measure appear in Table 5 .

Pairwise agreement by F-measure was fairly consistent between the groupings, with overall agreement ranging between 0.685 to 0.752 . Inconsistent use of the STRENGTH, STRENGTH_UNIT and REFILL tags lowered their agreement levels. The agreement levels for the INSTRUCTION tag were also predictably low, as INSTRUCTION is a catch-all tag for capturing patient instructions not captured elsewhere. Most of the other tags had relatively high agreement levels.

\section{Label and span accuracy}

Table 6 shows precision, recall, and f-measure scores for conditional random field modeling experiments for tag labels and spans. We report three experiments, one where the modeling is biased towards high precision scores, one biasing high recall scores, and a balanced run. The balanced run performs best overall, with an overall f-measure score of 0.748 , and a narrow spread of precision and recall. Training with a bias towards precision boosts precision significantly (to 0.996), at the expense of recall (0.407). Surprisingly, training with a bias towards recall fails to boost recall $(0.726)$ but does lower precision (0.651). Overall modeling results for labels and
Table 5 Pairwise agreement F-measures by annotation group and tag

\begin{tabular}{llllll}
\hline Tag & G1 & G2 & G3 & G4 & Average \\
\hline Dispense & 0.800 & 0.606 & 0.750 & 0.750 & 0.727 \\
Dispense_unit & 0.870 & 0.611 & 0.421 & 0.556 & 0.614 \\
Doseamount & 0.786 & 0.845 & 0.779 & 0.841 & 0.813 \\
Doseamount_unit & 0.828 & 0.810 & 0.707 & 0.822 & 0.792 \\
Doseform & 0.943 & 0.917 & 0.939 & 0.950 & 0.937 \\
Duration & 0.868 & 0.738 & 0.647 & 0.824 & 0.769 \\
Duration_unit & 0.916 & 0.779 & 0.702 & 0.868 & 0.816 \\
FREQ & 0.919 & 0.897 & 0.850 & 0.883 & 0.887 \\
Indication & 0.729 & 0.729 & 0.795 & 0.698 & 0.738 \\
Instruction & 0.260 & 0.294 & 0.229 & 0.381 & 0.291 \\
Medication & 0.861 & 0.528 & 0.769 & 0.723 & 0.721 \\
PRN & 0.786 & 0.977 & 0.800 & 0.920 & 0.870 \\
Refill & 0.000 & 0.000 & 0.500 & 0.000 & 0.125 \\
Route & 0.689 & 0.674 & 0.713 & 0.520 & 0.649 \\
Strength & 0.154 & 0.476 & 0.500 & 0.000 & 0.283 \\
Strength_unit & 0.167 & 0.421 & 0.615 & 0.000 & 0.301 \\
Sub_status & 0.609 & 0.786 & 0.929 & 0.462 & 0.696 \\
Take & 0.955 & 0.830 & 0.847 & 0.928 & 0.890 \\
Timing & 0.774 & 0.504 & 0.721 & 0.609 & 0.652 \\
Overall & 0.752 & 0.685 & 0.720 & 0.747 & 0.726 \\
\hline
\end{tabular}

spans are encouraging, but show substantial room for improvement, particularly for the lower-frequency labels.

\section{Attribute accuracy}

Table 7 shows accuracy results for modeling attribute values of various types. These experiments involved predicting attribute values for manually annotated span labels (thus there is no compounding of span prediction errors with attribute prediction errors). For choice attributes the Levenshtein Fallback and Classifier methods perform best (side being a notable exception where Literal Fallback outperforms Levenshtein Fallback). For the attributes of FREQ and the attributes of TIMING both the Hybrid and Classifier methods do quite well, with most accuracy scores in the 0.9-1.0 range. For the numeric attributes the Normalized method outperforms the Un-normalized method, by a large margin for some attributes. The exception is to_amt, where the Un-normalized method is slightly better. Finally, for Boolean attributes the Classifier method outperforms the Baseline method.

\section{Discussion}

The results of our annotation efforts show that it is possible to create a detailed annotation schema that captures a variety of information about prescription 
Table 6 Precision, recall, and F-measure results for labels and spans

\begin{tabular}{|c|c|c|c|c|c|c|c|c|c|c|}
\hline \multirow[t]{2}{*}{ Tag } & \multirow[t]{2}{*}{ \# train } & \multicolumn{3}{|c|}{ Balanced P \& R } & \multicolumn{3}{|c|}{ Precision Bias } & \multicolumn{3}{|c|}{ Recall Bias } \\
\hline & & $P$ & $\mathrm{R}$ & $F$ & $P$ & $R$ & $\mathrm{~F}$ & $P$ & $\mathrm{R}$ & $F$ \\
\hline Dispense & 46 & 1.000 & 0.333 & 0.500 & 1.000 & 0.364 & 0.222 & 1.000 & 0.333 & 0.500 \\
\hline Dispense_unit & 73 & 0.857 & 0.316 & 0.462 & 1.000 & 0.348 & 0.211 & 1.000 & 0.316 & 0.480 \\
\hline Doseamount & 221 & 0.900 & 0.614 & 0.730 & 1.000 & 0.275 & 0.159 & 1.000 & 0.500 & 0.667 \\
\hline Doseamount_unit & 202 & 0.921 & 0.761 & 0.833 & 1.000 & 0.386 & 0.239 & 1.000 & 0.630 & 0.773 \\
\hline Doseform & 533 & 0.975 & 0.826 & 0.895 & 1.000 & 0.769 & 0.625 & 0.981 & 0.736 & 0.841 \\
\hline Duration & 205 & 1.000 & 0.754 & 0.860 & 1.000 & 0.659 & 0.492 & 1.000 & 0.590 & 0.742 \\
\hline Duration_unit & 193 & 1.000 & 0.772 & 0.871 & 1.000 & 0.705 & 0.544 & 1.000 & 0.579 & 0.733 \\
\hline FREQ & 1329 & 0.978 & 0.735 & 0.840 & 0.990 & 0.683 & 0.522 & 0.977 & 0.700 & 0.816 \\
\hline Indication & 319 & 0.879 & 0.296 & 0.443 & 1.000 & 0.310 & 0.184 & 0.852 & 0.235 & 0.368 \\
\hline Instruction & 4075 & 0.602 & 0.892 & 0.719 & 1.000 & 0.068 & 0.035 & 0.510 & 0.928 & 0.658 \\
\hline Medication & 145 & 1.000 & 0.103 & 0.188 & 1.000 & 0.000 & 0.000 & 1.000 & 0.103 & 0.188 \\
\hline PRN & 232 & 1.000 & 0.855 & 0.922 & 1.000 & 0.804 & 0.673 & 1.000 & 0.782 & 0.878 \\
\hline Refill & 70 & 1.000 & 0.000 & 0.000 & 1.000 & 0.000 & 0.000 & 1.000 & 0.000 & 0.000 \\
\hline Route & 591 & 0.947 & 0.423 & 0.584 & 1.000 & 0.408 & 0.256 & 0.884 & 0.363 & 0.515 \\
\hline Strength & 33 & 1.000 & 0.000 & 0.000 & 1.000 & 0.000 & 0.000 & 1.000 & 0.000 & 0.000 \\
\hline Strength_unit & 34 & 1.000 & 0.000 & 0.000 & 1.000 & 0.000 & 0.000 & 1.000 & 0.000 & 0.000 \\
\hline Sub_status & 112 & 0.929 & 0.500 & 0.650 & 1.000 & 0.000 & 0.000 & 1.000 & 0.462 & 0.632 \\
\hline Take & 774 & 0.952 & 0.855 & 0.901 & 0.993 & 0.726 & 0.573 & 0.962 & 0.761 & 0.850 \\
\hline Timing & 1359 & 0.979 & 0.634 & 0.770 & 1.000 & 0.506 & 0.339 & 0.908 & 0.564 & 0.696 \\
\hline Overall & 10,546 & 0.743 & 0.753 & 0.748 & 0.996 & 0.407 & 0.256 & 0.651 & 0.726 & 0.687 \\
\hline
\end{tabular}

For each tag, the highest performing f-measure is presented in boldface

directions in a structured way. Our pairwise agreement levels show that most of the tags in this schema can be applied in a consistent manner. The agreement levels show room for improvement, and point to the need to adjudicate a gold standard (which we did). There is always a tradeoff between the complexity of an annotation schema and the consistency with which it can be applied, as reflected in pairwise agreement numbers. The lower agreement numbers of the STRENGTH and STRENGTH_UNIT tags may be a result of their confusability with DOSEAMOUNT and DOSEAMOUNT_UNIT. These two sets of tags have clearly different uses, but capture similar information. In a complex annotation task such as ours these distinctions can become too subtle to apply consistently, and the more frequently occurring tags (DOSEAMOUNT and DOSEAMOUNT_UNIT in this comparison) can become the default in an annotator's mind for particular text strings.

One of the lower performing tags in our label and span modeling is MEDICATION. Of the 26 MEDICATIONs in the test corpus, just three were correctly identified by label and span in the Balanced model. Nine were assigned an INSTRUCTION tag (and often a longer span) by the model, and 14 were missed entirely. This result is unsurprising. MEDICATION strings vary greatly, as do INSTRUCTION strings, and are very sparse in this corpus, as medications often appear only in the structured data and not in the patient prescription regimen string. The methods in this study relied solely on our small training set, whereas any system intended for production use should rely on a medication name vocabulary (such as RxNorm) as an additional source of information. Our attribute modeling experiments show that there are methods available to assign attributes automatically at a high level of accuracy. However, the best-performing methods differ for different attribute types. The Classifier methods tend to perform at or near the top for all classes of attributes, save numeric, which we did not model with any classification method.

Our study is limited in that it describes an annotation schema developed over a single corpus of prescription regimens. As there was no earlier effort to build on, development of the schema was a labor-intensive task, involving several rounds of pilot annotation and refinement of the schema. The schema has not been validated against a second corpus from a different source; this would be a valuable direction for future work.

Previous related work in de-identification has shown that the labor needed to apply a schema to a corpus can be significantly reduced by iteratively applying preliminary 
Table 7 Accuracy results for tag attributes

\begin{tabular}{|c|c|c|c|c|}
\hline Choice Attributes & Baseline & Literal Fallback & Levenshtein Fallback & Classifier \\
\hline form & 0.597 & 0.944 & 0.965 & 0.944 \\
\hline route & 0.339 & 0.667 & 0.738 & 0.887 \\
\hline side & 0.792 & 0.935 & 0.173 & 0.935 \\
\hline unit & 0.477 & 0.831 & 0.869 & 0.823 \\
\hline Attributes of FREQ & Baseline & Hybrid & Classifier & \\
\hline every & 0.808 & 0.984 & 0.981 & \\
\hline to_every & 0.954 & 1.000 & 0.986 & \\
\hline times_per & 0.570 & 0.927 & 0.900 & \\
\hline to_times_per & 0.876 & 0.949 & 0.954 & \\
\hline unit & 0.722 & 0.976 & 0.976 & \\
\hline Attributes of TIMING & Baseline & Hybrid & Classifier & \\
\hline direction & 0.396 & 0.990 & 0.990 & \\
\hline event & 0.406 & 0.919 & 0.919 & \\
\hline offset & 0.480 & 0.883 & 0.742 & \\
\hline to_offset & 0.866 & 0.950 & 0.950 & \\
\hline offset_unit & 0.430 & 0.826 & 0.826 & \\
\hline Numeric Attributes & Un-normalized & Normalized & & \\
\hline amt & 0.160 & 0.927 & & \\
\hline to_amt & 0.882 & 0.857 & & \\
\hline num & 0.115 & 0.885 & & \\
\hline to_num & 0.836 & 0.885 & & \\
\hline quantity & 0.889 & 1.000 & & \\
\hline to_quantity & 1.000 & 1.000 & & \\
\hline Boolean Attributes & Baseline & Classifier & & \\
\hline refill & 0.333 & 1.000 & & \\
\hline subst & 0.500 & 0.615 & & \\
\hline
\end{tabular}

machine-learned models to unseen data as pre-taggers [29]. By doing this, the annotation task becomes a correction task (inspecting and correcting the output of the preliminary models), which has been shown to speed-up model and corpus development [30]. A logical next step for this work is to apply these tag-a-little, learn-a-little principles to bootstrap the development of an annotated prescription regimen corpus from a second source, to validate our approach.

\section{Conclusions}

Through an annotation development effort, we have demonstrated a method for capturing structured data from prescription regimen strings, and have shown that the schema can be applied manually with high accuracy for many tag label types. We have further shown that conditional random field modeling techniques can apply tag labels to text spans with similar accuracy levels in this corpus, and that various modeling techniques can correctly set the attributes of these tags at high accuracy. Future work can address the applicability of these techniques to other corpora, and explore using tag-a-little, learn-a-little iterative model and corpus development to reduce the labor needed to create annotated corpora of prescription regimens.

The strings in our corpus are textual representations of prescription regimens, complete with errors. By structuring the textual representation through annotation, the text can be compared against the pharmacist-entered structured data (through one-to-one data structure mapping in the case of FHIR-compliant pharmacy data), offering an opportunity to detect and correct discrepancies.

TranScriptML is a richer representation of medication regimen information than those used in previous natural language annotation efforts, and is consistent with emerging standards for representation of structured data in the same domain. We hope these standards will encourage compatibility between clinical NLP tools and the Electronic Health Record (EHR) software ecosystem. For these reasons, we are releasing our annotation schema and guidelines alongside this report, and urge that TranScriptML or compatible representations be used in future corpus development. 


\section{Additional files}

Additional file 1: TranScriptMLAnnotationGuidelines.docx (MS Word document). TranscriptML Annotation Guidelines. Guidelines for applying the TranscriptML annotation schema to textual prescription regimens. (DOCX 56 kb)

Additional file 2: TranscriptAnnotationTask.xml (XML file). TranscriptML Task Definition. XML file defining the Transcript task for use with the MITRE Annotation Toolkit. (XML $19 \mathrm{~kb}$ )

\section{Acknowledgements}

The authors are grateful to Dr. Alexander Turchin and Dr. Matvey Palchuk (Harvard Medical School) for granting us access to the data used in this work, to Lewis Blackwell II (MITRE) for assistance in preparing the manuscript, and to Dr. Lynette Hirschman, John Burger, and Art Hamerschlag (MITRE) for their review and advice during our research process.

\section{Funding}

This research was funded by MITRE's internal research program.

\section{Availability of data and materials}

The annotation guidelines and MITRE Annotation Toolkit task definition XML file are included as Additional files 1 and 2. The MITRE Annotation Toolkit is available at http://mat-annotation.sourceforge.net/.

\section{Authors' contributions}

JA, CC, MK, and DTK contributed to the design of the annotation schema, and the annotation effort itself. JA, SB, and DTK contributed to the modeling and analysis effort. All authors contributed to the preparation of the manuscript.

\section{Ethics approval and consent to participate}

The data used in this study were de-identified and provided under a data use agreement. No ethics approval of consent to participate was required.

\section{Consent for publication}

The data used in this study were de-identified and provided under a data use agreement. No consent for publication is required.

\section{Competing interests}

The authors declare that they have no competing interests.

\section{Publisher's Note}

Springer Nature remains neutral with regard to jurisdictional claims in published maps and institutional affiliations.

\section{Received: 27 February 2017 Accepted: 7 May 2019}

Published online: 31 May 2019

\section{References}

1. Evans DA, Brownlow ND, Hersh WR, Campbell EM. Automating concept identification in the electronic medical record: an experiment in extracting dosage information. Proc AMIA Annu Fall Symp. 1996:388-92.

2. Friedman C, Shagina L, Lussier Y, Hripcsak G. Automated encoding of clinical documents based on natural language processing. J Am Med Inform Assoc. 2004;11:392-402.

3. Cimino JJ, Bright TJ, Li J. Medication reconciliation using natural language processing and controlled terminologies. Stud health Technol inform, vol. 129; 2007. p. 679-83.

4. Gold S, Elhadad N, Zhu X, Cimino JJ, Hripcsak G. Extracting structured medication event information from discharge summaries. AMIA Annual Symposium proceedings / AMIA Symposium AMIA Symposium American Medical Informatics Association. 2008;2008:237-41.

5. Jagannathan V, Mullett CJ, Arbogast JG, Halbritter KA, Yellapragada D, Regulapati S, et al. Assessment of commercial NLP engines for medication information extraction from dictated clinical notes. Int J Med Inform. 2009; 78:284-91

6. Uzuner Ö, Solti I, Cadag E. Extracting medication information from clinical text. J Am Med Inform Assoc. 2010;17:514-8.
7. Tikk D, Solt I. Improving textual medication extraction using combined conditional random fields and rule-based systems. J Am Med Inform Assoc. 2010;17:540-4.

8. Deleger L, Grouin C, Zweigenbaum P. Extracting medical information from narrative patient records: the case of medication-related information. J Am Med Inform Assoc. 2010;17:555-8.

9. Mork JG, Bodenreider O, Demner-Fushman D, Dogan RI, Lang F-M, Lu Z, et al. Extracting Rx information from clinical narrative. J Am Med Inform Assoc. 2010:17:536-9.

10. Yang $\mathrm{H}$. Automatic extraction of medication information from medical discharge summaries. J Am Med Inform Assoc. 2010:17:545-8.

11. Hamon T, Grabar N. Linguistic approach for identification of medication names and related information in clinical narratives. J Am Med Inform Assoc. 2010;17:549-54

12. Spasic I, Sarafraz F, Keane JA, Nenadic G. Medication information extraction with linguistic pattern matching and semantic rules. J Am Med Inform Assoc. 2010;17:532-5.

13. Doan S, Bastarache L, Klimkowski S, Denny JC, Xu H. Vanderbilt's system for medication extraction. Proceedings of the Third i2b2 ..; 2009.

14. Patrick J, Li M. High accuracy information extraction of medication information from clinical notes: 2009 i2b2 medication extraction challenge. J Am Med Inform Assoc. 2010;17:524-7.

15. Li Z, Liu F, Antieau L, Cao Y, Yu H. Lancet: a high precision medication event extraction system for clinical text. J Am Med Inform Assoc. 2010;17:563-7.

16. Meystre SM, Thibault J, Shen S, Hurdle JF, South BR. Textractor: a hybrid system for medications and reason for their prescription extraction from clinical text documents. J Am Med Inform Assoc. 2010;17:559-62.

17. Wang Y, Steinhubl SR, Defilippi C, Ng K, Ebadollahi S, Stewart WF, et al. Prescription extraction from clinical notes: towards automating EMR medication reconciliation. AMIA Jt Summits Transl Sci Proc American Medical Informatics Association. 2015:2015:188-93.

18. Sohn S, Clark C, Halgrim SR, Murphy SP, Chute CG, Liu H. MedXN: an open source medication extraction and normalization tool for clinical text. J Am Med Inform Assoc. 2014;21:858-65.

19. Selden CR, Humphreys BL. Unified medical language system: DIANE Publishing; 1997.

20. Liu S, Ma W, Moore R, Ganesan V, Nelson S. RxNorm: prescription for electronic drug information exchange. IT professional. IEEE Educational Activities Department. 2005;7:17-23.

21. Deleger L, Li Q, Lingren T, Kaiser M, Molnar K, Stoutenborough $L$, et al. Building gold standard corpora for medical natural language processing tasks. AMIA Annual Symposium proceedings / AMIA Symposium AMIA Symposium. 2012;2012:144-53

22. Savova G, Styler W, Albright D, Palmer M, Harris D. SHARP template annotations: guidelines [internet]. 2012. Available from: http://informatics. mayo.edu/sharp/images/e/e2/SHARP_Template_Annotation_Guidelines task1.5.3_1.6.3.pdf

23. Belknap S, Freund E, Frid N, Granillo E, Keating H, Li Z, et al. The Annotation Guideline Manual: Extracting Adverse Drug Event Information from Clinical Narratives in Electronic Medical Records [Internet]. 2015 [cited 2016 Aug 26]. pp. 1-60. Available from: https://manualzz.com/doc/7543004/theannotation-guideline-manual--extracting.

24. Bender D, Sartipi K. HL7 FHIR: an agile and RESTful approach to healthcare information exchange. IEEE 26th International Symposium on ComputerBased Medical Systems (CBMS) IEEE. 2013:2013:326-31.

25. HL7 FHIR Documentation: MedicationOrder [Internet]. [cited 2016 Aug 26] Available from: http://hl7.org/fhir/DSTU2/medicationorder.html.

26. Aberdeen J, Bayer S, Yeniterzi R, Wellner B, Clark C, Hanauer D, et al. The MITRE identification scrubber toolkit: design, training, and assessment. Int J Med Inform. 2010;79:849-59.

27. Wellner B. Sequence models and ranking methods for discourse parsing [Ph.D. Dissertation]. Waltham, MA: Brandeis University; 2009.

28. Levenshtein VI. Binary codes capable of correcting deletions, insertions, and reversals. Soviet physics doklady; 1966.

29. Hanauer D, Aberdeen J, Bayer S, Wellner B, Clark C, Zheng K, et al. Bootstrapping a de-identification system for narrative patient records: costperformance tradeoffs. Int J Med Inform. 2013;82:821-31.

30. Day D, Aberdeen J, Hirschman L, Kozierok R, Robinson P, Vilain M. Mixedinitiative development of language processing systems. Fifth Conference on Applied Natural Language Processing Morristown, NJ, USA: Association for Computational Linguistics. 1997:348-55. 